\title{
Downregulation of miR-221/222 sensitizes glioma cells to temozolomide by regulating apoptosis independently of p53 status
}

\author{
LINGCHAO CHEN $^{1 *}$, JUNXIA ZHANG ${ }^{2,3^{*}}$, LEI HAN ${ }^{2,3}$, ANLING ZHANG $^{2,3}$, CHUNZHI ZHANG $^{2,3}$, \\ YONGRI ZHENG ${ }^{1}$, TAO JIANG ${ }^{4}$, PEIYU PU ${ }^{2,3}$, CHUANLU JIANG ${ }^{1}$ and CHUNSHENG KANG ${ }^{2,3}$ \\ ${ }^{1}$ Department of Neurosurgery, The Second Affiliated Hospital of Harbin Medical University, Harbin 150086; \\ ${ }^{2}$ Department of Neurosurgery, Tianjin Medical University General Hospital, Laboratory of Neuro-Oncology, \\ Tianjin Neurological Institute, Tianjin 300060; ${ }^{3}$ Key laboratory of Neurotrauma, Variation and \\ Regeneration, Ministry of Education and Tianjin Municipal Government, Tianjin 300060; ${ }^{4}$ Department \\ of Neurosurgery, Tiantan Hospital, Capital Medical University, Beijing 100050, P.R. China
}

Received August 25, 2011; Accepted October 7, 2011

DOI: $10.3892 / o r .2011 .1535$

\begin{abstract}
A previous study showed that miR-221/222 can regulate cell apoptosis. p53 is a well known tumor suppressor which can influence the chemosensitivity of glioma cells. However, the effect of miR-221/222 in gliomas with different p53 status is unknown. Here, we demostrate that knockdown of miR-221/222 increases apoptosis in human gliomas of different p53 types (U251 cells, p53 mutant-type; LN308 cells, p53 null-type; and U87 cells, p53 wild-type). Furthermore, the effect of miR-221/22 caused no change of p53 expression in the glioma cells studied. In addition, when a specific siRNA against p53 was employed in U87 cells, no attenuation of apoptosis was found after knockdown of miR-221/222. Importantly, we found that As-miR-221/222-treated cells increased expression of Bax, cytochrome c, Apaf-1 and cleaved-caspase-3. Our results showed that low expression of miR-221/222 sensitized glioma cells to temozolomide (TMZ); in addition, ectopic expression of PUMA by pcDNA-PUMA had a similar effect. Taken together, our study indicates that downregulated miR-221/222 can sensitize glioma cells to TMZ by regulating apoptosis independently of p53 status.
\end{abstract}

Correspondence to: Dr Chuanlu Jiang, Department of Neurosurgery, The Second Affiliated Hospital of Harbin Medical University, Harbin 150086, P.R. China

E-mail: jc16688@163.com

Dr Chunsheng Kang, Laboratory of Neuro-Oncology, Tianjin Neurological Institute, 152 Ahshan Road, Heping, Tianjin 300052, P.R. China

E-mail: kang97061@yahoo.com

*Contributed equally

Key words: miR-221/222, glioblastoma, temozolomide, p53

\section{Introduction}

Glioblastoma, the most aggressive type of tumor arising in the central nervous system (CNS), is considered to be one of the deadliest of human cancers. Despite the advances in surgery, radiation therapy, and chemotherapy, the 1-year overall survival rate is less than $30 \%$, and the median survival time of patients with high-grade glioma is only approximately 15 months $(1,2)$. To develop more optimized and effective treatment strategies for glioblastomas, it is critical to gain deeper understanding of the molecular mechanisms underlying gliomagenesis and to identify targets for therapeutic intervention $(3,4)$.

MicroRNAs are small regulatory RNA molecules that in recent years have been identified in the progression of various cancers and proposed as novel targets for anticancer therapies $(5,6)$. By negatively regulating their mRNA targets to either degradation or translational repression, they can act as both tumor suppressors and oncogenes (7). Recent studies showed frequent deregulation of miR-221/222 in glioblastoma and astrocytoma cell lines (8). Overexpression of miR-221/222 increased glioma cell proliferation and invasion in vitro and induced glioma growth in a subcutaneous mouse model (9). In addition, blockade of miR-221/222 cluster suppressed human glioma cell growth, suggesting the cosuppression of miR-221/222 cluster might be a potential therapeutic strategy in glioma (10). Our previous study demostrated that miR-221/222 can directly target PUMA inducing apoptosis (11). p53, an upstream gene of PUMA, is a well known tumor-suppressor. However, the role of miR-221/222 in glioma of different p53 status is not well elucidated.

In this study, we identified the involvement of miR-221/222 in mediating apoptosis in glioma regardless of p53 status. Furthermore, we confirmed that low expression of miR-221/222 sensitizes glioma cells to the alkylating agent TMZ partly due to upregulation of PUMA. 


\section{Materials and methods}

Cell culture and treatment. Human glioblastoma cells (LN308, U251 and U87) were obtained from the China Academia Sinica Cell Repository, Shanghai, China. The cells were maintained in Dulbecco's modified Eagle's medium (DMEM) (Gibco) supplemented with $10 \%$ fetal bovine serum (Gibco), $2 \mathrm{mM}$ glutamine (Sigma), $100 \mu \mathrm{g} / \mathrm{ml}$ penicillin (Sigma), and $100 \mu \mathrm{g} / \mathrm{ml}$ streptomycin (Sigma), and incubated at $37^{\circ} \mathrm{C}$ with $5 \% \mathrm{CO}_{2}$. Cell transfection were used Lipofectamine 2000 (Invitrogen) following the manufacturer's instructions. TMZ was purchased from Sigma, and was dissolved in DMSO.

Plasmids production and oligonucleotides. The pGL3WT-PUMA-3'UTR-Luc reporter was created by ligation of PCR products of 3'UTR of PUMA into the $X b a$ I site of the pGL3 control vector (Promega, USA). The primers for PCR amplification are: PUMA-3'UTR forward: 5'-TCA TGA ATTC GCC CCT CCC ACC TCC TGA CAC CCT GGC CAG CGC GGG GGA CTT TCT CTG C-3' and PUMA3'UTR reverse: 5'-CGC CCC CGG GAC AGG CAG GGC TGG GAG TCC AGT ATG CTA CAT GGT GCA GAG AAA GTC CC-3'. The pGL3-MUT-PUMA-3'UTR-Luc reporter was generated from pGL3-WT-PUMA-3'TR-Luc reporter by deleting the binding site for miR-221/222. HA-tagged wild-type PUMA in pCDNA3 was obtained from Volgelstein (12). The 2'-OMe-oligonucleotides were chemically synthesized and purified by GenePharma Co., Ltd. (Shanghai, China). The sequences are: 2'-OMe-As-miR-221 (As-miR-221), 5'-AGCUACAUUGUCUGCUGGGUUUC-3'; 2'-OMe-As-miR-222 (As-miR-222), 5'-AGCUACAUCUG GCUACUGGGU-3'. Scrambled RNA and p53 siRNA were purchased from GenePharma Co., Ltd. The p53 siRNA sequences are: sense 5'-GACUCCAGUGGUAAUCUACTT-3', antisense 5'-GUAGAUUACCACUGGAGUCTT-3'.

RNA extraction and RT real-time PCR analysis. Total RNA was extracted using TRIzol (Invitrogen), according to the manufacturer's instructions. After a sufficient amount of cDNA was obtained, PCR amplification was performed using a real-time PCR cycler (7500 ABI, USA). Expressions of mature miR-221/222 was quantified by miR-qRT PCR using Hairpin-it ${ }^{\mathrm{TM}}$ miRNA qPCR Quantitation Kit 313 (GenePharma Co., Ltd). The level of miRNA expression was measured using $\mathrm{Ct}$ (threshold cycle). The $\Delta \Delta \mathrm{Ct}$ method for relative quantitation of gene expression was used to determine miRNA expression levels. The $\Delta \mathrm{Ct}$ was calculated by subtracting the $\mathrm{Ct}$ of $U 6$ RNA from the $\mathrm{Ct}$ of the miRNA of interest. The $\Delta \Delta \mathrm{Ct}$ was calculated by subtracting the $\Delta \mathrm{Ct}$ of the reference sample from the $\Delta \mathrm{Ct}$ of each sample. Fold change was generated using the equation $2^{-\Delta \Delta \mathrm{Ct}}$. The Taqman MicroRNA Assays for U6 RNA (RNU6B, P/N: 4373381; Applied Biosystems) was used to normalize the relative abundance of miRNA. The results of real-time PCR were presented as the ratio between the selected genes and U6 transcripts.

Western blot analysis and luciferase reporter assay. LN308, U251 and U87 cells were lysed in 1\% Nonidet P-40 lysis buffer after transfection. Homogenates were clarified by centrifugation at $20,000 \times \mathrm{g}$ for $15 \mathrm{~min}$ at $4^{\circ} \mathrm{C}$, and protein concentration was measured by the Lowry method. SDS-PAGE was performed on $40 \mu \mathrm{g}$ of protein from each sample, gels were transferred to PVDF membranes (Millipore, USA) and incubated with primary antibodies against p53, PUMA, Bax, Bcl-2, cytochrome c, Apaf-1, caspase-3 and cleaved caspase-3 (1:1000 dilution, Santa Cruz, USA), followed by incubation with an HRP-conjugated secondary antibody (1:1000 dilution, Zymed, San Diego, CA). After washing with stripping buffer, the membrane was reprobed with antibody against GAPDH. For reporter assay, cells were cultured in 96-well plates and transfected with pGL3-PUMA-3'UTR-Luc or pGL3-PUMAmutant-3'UTR-Luc, and As-miR-221 and/or As-miR-221. Following 48-h incubation, luciferase activity was measured using a dual-luciferase reporter system (Promega). Each transfection was repeated twice in triplicate.

Apoptosis. Forty-eight hours after transfection, apoptosis in cultured cells was evaluated with Annexin V labeling and caspase-3/7 activity. For the Annexin V assay, an Annexin V-FITC labeled Apoptosis Detection Kit (Abcam) was used according to the manufacturer's protocol. Caspase-3/7 activity was measured using caspase-Glo 3/7 reagent (Promega).

Statistical analysis. Statistical evaluation for data analysis was determined by t-test. Differences with $\mathrm{P}<0.05$ were considered statistically significant.

\section{Results}

Downregulation of miR-221/222 increases apoptosis. To explore the role of miR-221/222 in apoptosis, we used a loss-of-function antisense approach. As-miR-221/222 oligonucleotides were used to knock down miR-221/222 expression in different p53 status of glioma (U251 cells, p53 mutant-type; LN308 cells, p53 null-type; and U87 cells, p53 wild-type). RT real-time PCR results determined that the relative expression level of miR-221/222 in As-miR-221/222 treated U251, LN308 and U87 cells were successfully reduced compared with their control cells, respectively (Fig. 1A). Furthermore, Annexin V labeling revealed that knockdown of miR-221 and miR-222 significantly increased cell apoptosis compared to the cells treated with scramble oligonucleotide (Fig. 1B). As anticipated, caspase-3/7 activity was also considerably elevated in miR-221 and miR-222 knocked down cells (Fig. 1C). Moreover, Western blot assay displayed that pro-apoptotic protein PUMA expression was significantly upregulated while p53 expression was unchanged (Fig. 1D). These findings indicated that knockdown of miR-221/222 play an important role in initiation of apoptosis in glioma cell lines.

Downregulation of miR-221/222 mediated apoptosis independent of p53. Next, we chose a p53 wild-type U87 cell line treated by 553 siRNA to detect whether they induced apoptosis by AS-miR-221/222. The mRNA and protein levels of p53 were successfully knocked down by p53 siRNA (Fig. 2A and B). After transfection, Annexin $\mathrm{V}$ labeling revealed that knockdown of miR-221/222 significantly increased cell apoptosis (Fig. 2C). Alternatively, caspase-3/7 activity was also considerably elevated (Fig. 2D). In additon, Western blot assay displayed that PUMA expression was significantly upregulated 
A

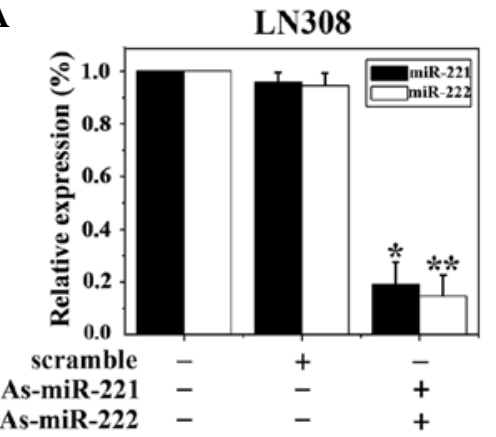

B

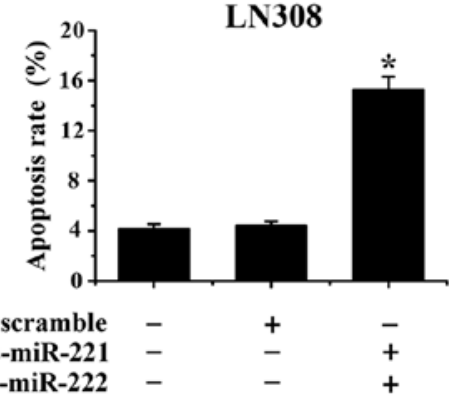

C

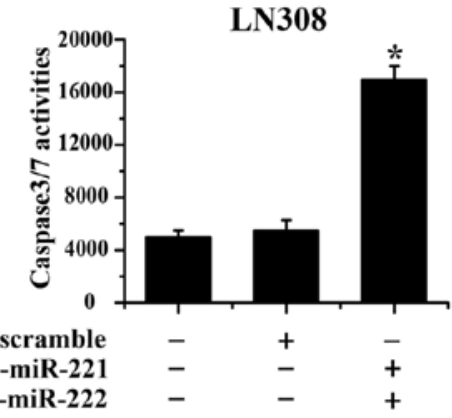

D

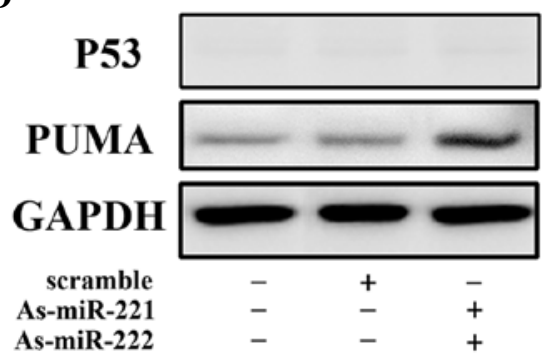

U251

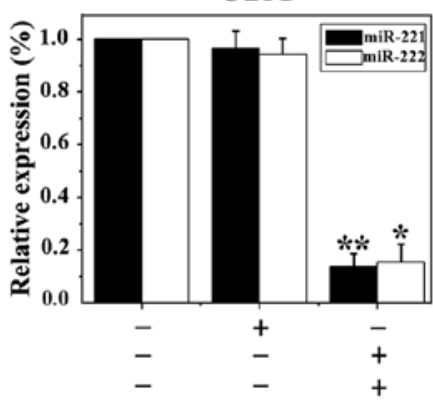

U251

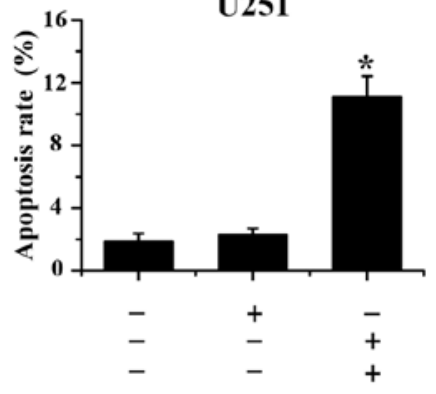

U251

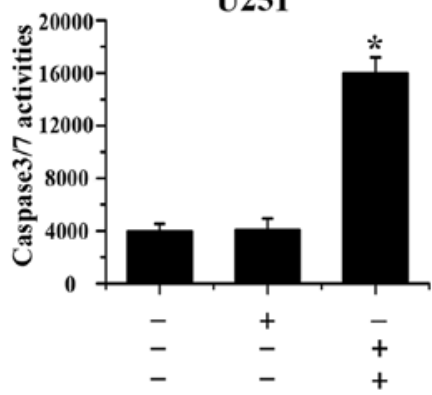

U251
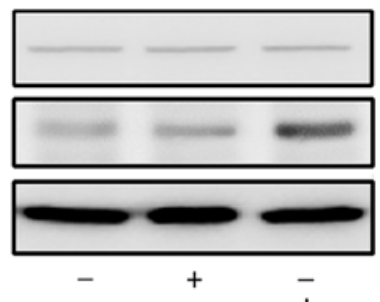

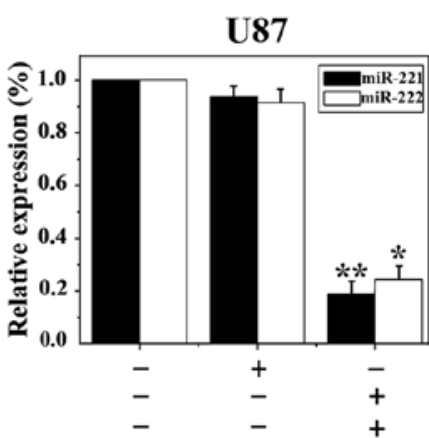

U87

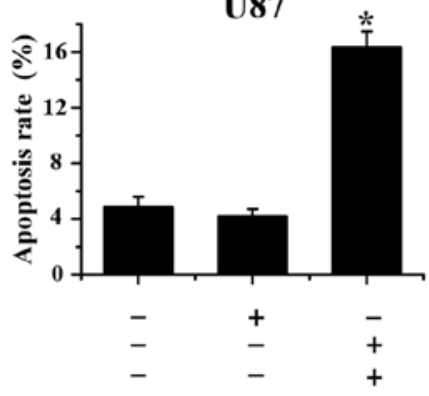

U87

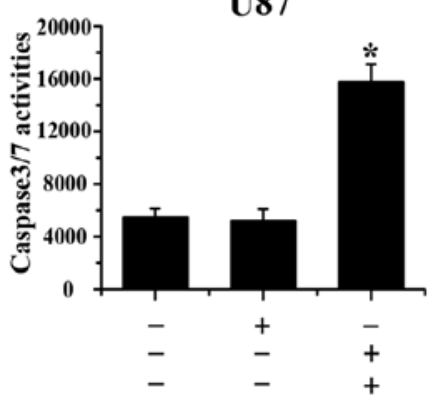

U87

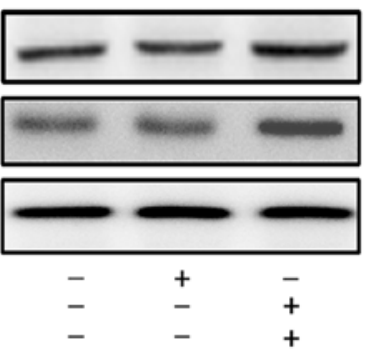

Figure 1. Downregulation of miR-221/222 increases apoptosis in glioma cells. (A) LN308, U251 and U87 cells were transfected with As-miR-221/222, and miR-221 and miR-222 expression levels were detected by real-time PCR. MiR-221/222 were decresed by miR-221/222 inhibitor-treated cells compared with control and scramble groups. (B) Annexin V analysis showed that LN308, U251 and U87 cells transfected with As-miR-221/222 displayed significantly more apoptosis than the other two groups. (C) A significant increase in caspase-3/7 activity was detected in LN308, U251 and U87 cells transfected with As-miR221/222. (D) LN308, U251 and U87 cells were transfected with As-miR-221/222, and p53 and PUMA protein level was detected by Western blot assay. GAPDH protein was regarded as endogenous normalizer. ${ }^{*} \mathrm{P}<0.05$ compared with control group, ${ }^{* *} \mathrm{P}<0.01$ compared with control group.

while the expression of p53 was unchanged in As-miR221/222 group (Fig. 2B). Combined with previous results, we concluded that downregulation of miR-221/222 increased PUMA mediated apoptosis independent of p53 status.

MiR-221/222 regulates the mitochondrial apoptosis pathway. To further analyze the mechanism of apoptosis mediated by miR-221/222, Western blot analysis showed low expression of miR-221/222 increased the expression of Bax, cytochrome c, Apaf- 1 and cleaved-caspase- 3 and decreased the expression of Bcl-2 in all groups (Fig. 3). Thus, our data indicated that apoptosis induced by As-miR-221/222 was activated in the mitochondrial apoptosis pathway.

PUMA is the target of miR-221/222. Using Pictar, we found 3'UTR of PUMA containing highly conserved putative miR-221 and miR-222 binding sites. To examine whether there is a direct regulation of PUMA mRNA by miR-221/-222, we cloned the 3'UTR region downstream of the luciferase open reading frame, and used this reporter construct to transfect 
A

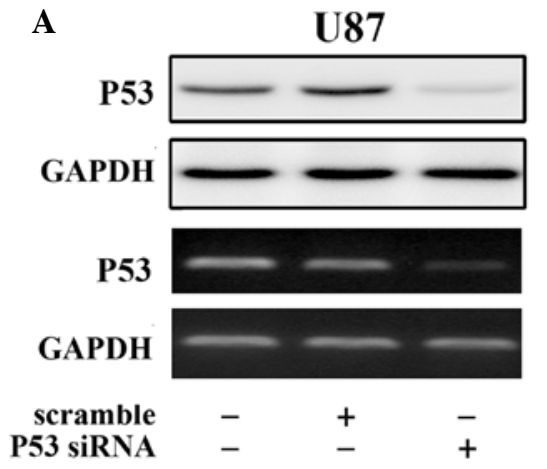

B

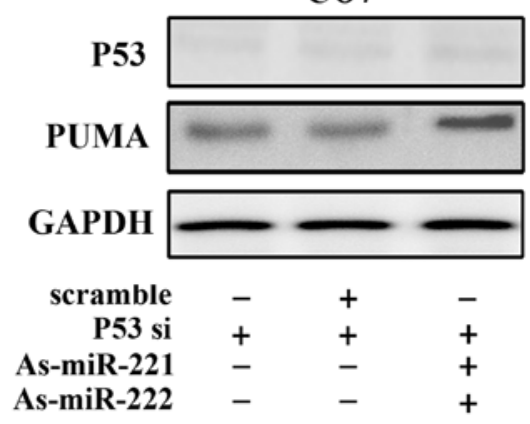

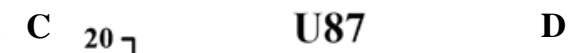

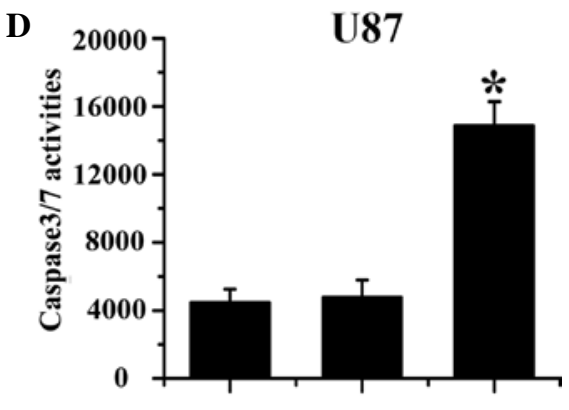

$\begin{array}{rrrr}\text { scramble } & - & + & - \\ \text { P53 si } & + & + & + \\ \text { As-miR-221 } & - & - & + \\ \text { As-miR-222 } & - & - & +\end{array}$

Figure 2. Downregulation of miR-221/222 increases apoptosis independent of p53 status. (A) p53 siRNA, followed transfected into U87 cells, eliciting near complete p53 mRNA and protein elimination by Western blot analysis and RT-PCR. (B) p53 siRNA treated U87 cells were transfected with As-miR-221/222, p53, PUMA expression levels were detected by Western blot assay. (C) Annexin V analysis showed that p53 siRNA treated U87 cells transfected with As-miR221/222 displayed significantly more apoptosis than control and scramble groups. (D) A significant increase in caspase-3/7 activity was detected in p53 siRNA treated U87 cells transfected with As-miR-221/222.

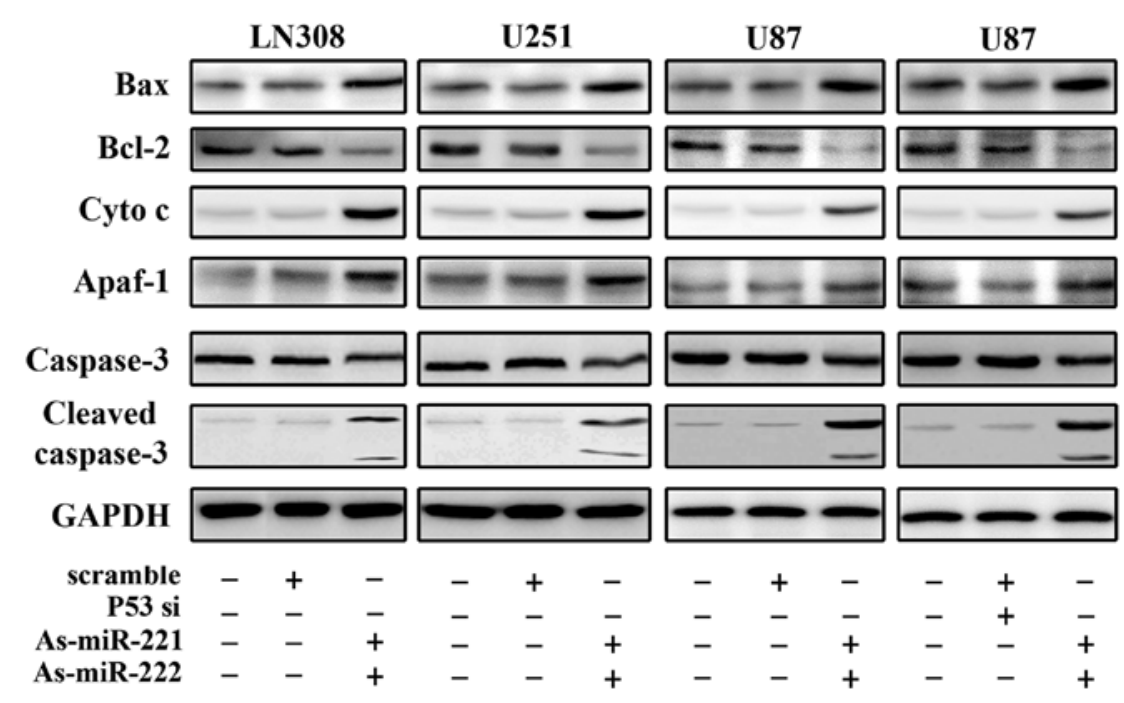

Figure 3. Impact of As-miR-221/222 on components of mitochondrail apoptosis pathway. All different p53 type glioma cells (U251 cells, p53 mutant-type; LN308 cells, p53 null-type; and U87 cells, p53 wild-type) and p53 siRNA treated U87 cells were treated with As-miR-221/222, as described in Materials and methods. Expression of Bax, Bcl-2, cytochrome c, Apaf-1, caspase-3, cleaved-caspase-3 and GAPDH was determined $48 \mathrm{~h}$ following treatment by Western blot analysis. Data are from one of three representative experiments.

U251, LN308 and U87 cells along with control or As-miR221/222. An increased relative luciferase activity was noted with As-miR-221/222 in all glioma cells (Fig. 4A), indicating that miR-221/222 can modulate gene expression directly at PUMA 3'UTR.
Low expression of miR-221/222 increases TMZ-induced cytotoxicity in glioma cells of different $p 53$ status. The glioma cells treated with TMZ (Fig. 4B) were depressed with miR-221/222 inhibitor. The AS-miR-221/222-induced potentiation of the effects of TMZ were recognized at a lower concentration 
A
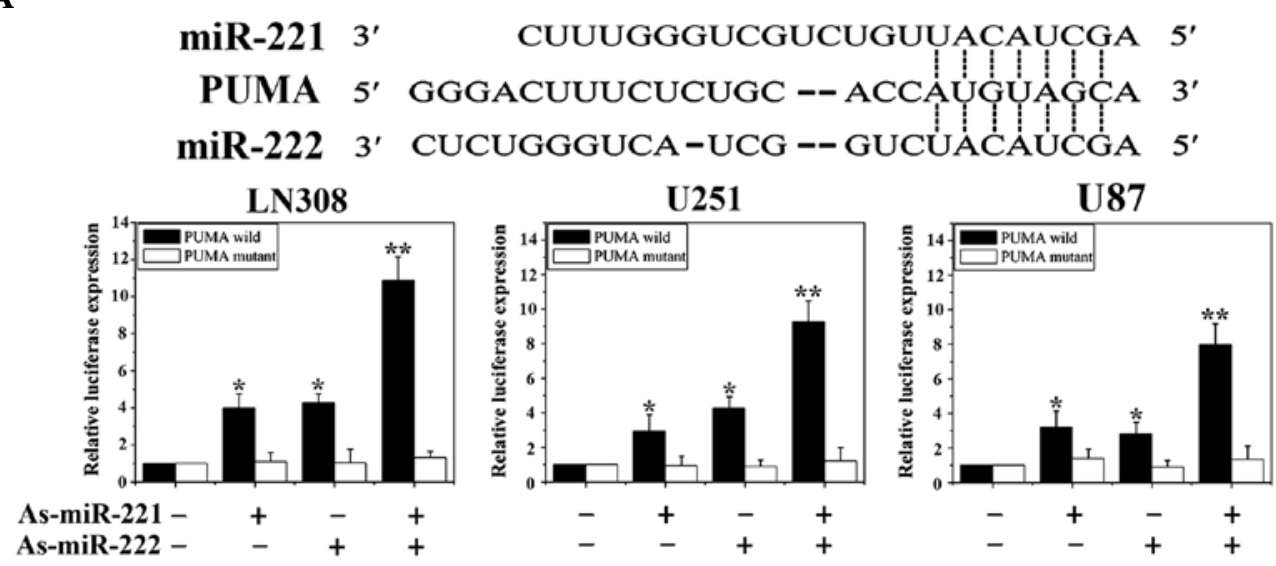

B
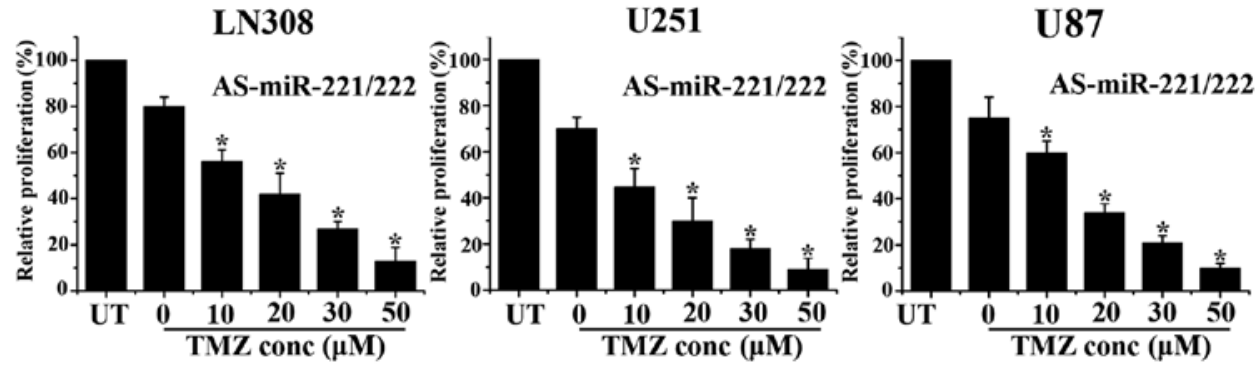

C

LN308

U251
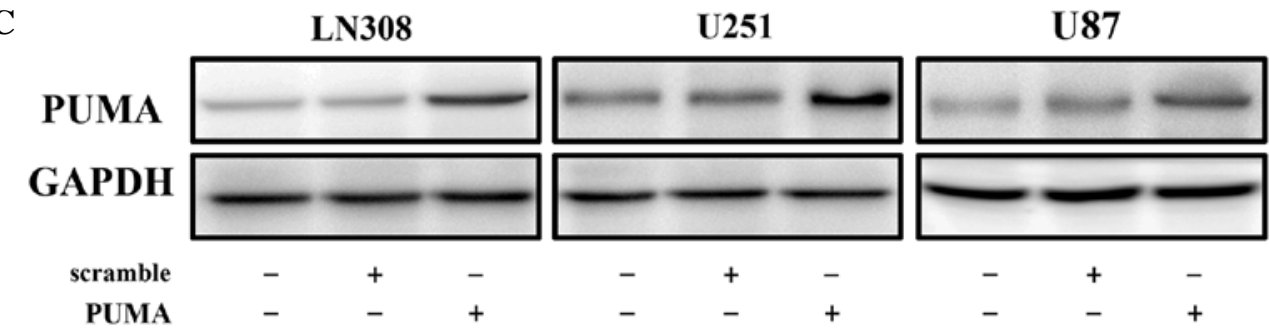

D
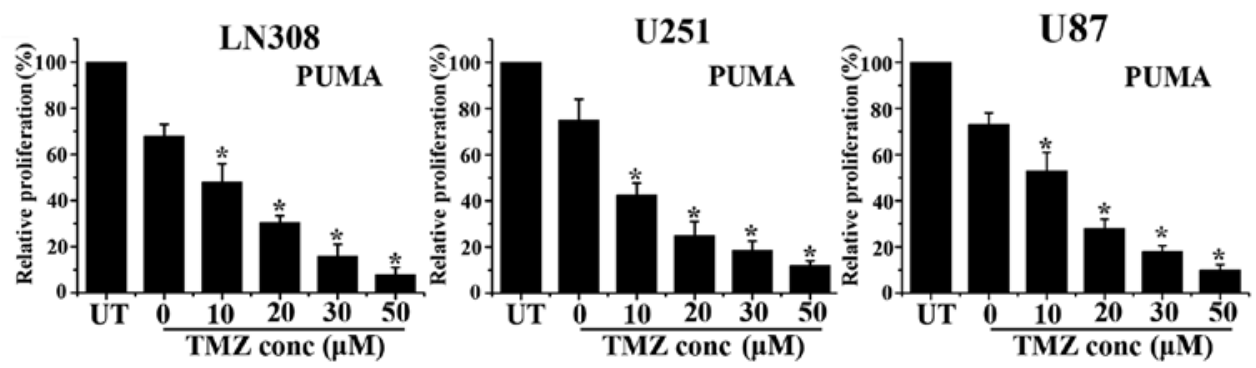

Figure 4. Low expression of miR-221/222 increases TMZ-induced cytotoxicity in glioma cells. (A) PUMA wild and muant luciferse reporter plasmids were transfected into LN308, U251 and U87 cells which were transfected with As-miR-221 and/or As-miR-222. Luciferase activity was determined 48 h after transfection. The ratio of normalized sensor to control luciferase activity is shown. ${ }^{*} \mathrm{P}<0.05$ compared with control group, ${ }^{* *} \mathrm{P}<0.01$ compared with control group. (B) Relative proliferation of LN308, U251 and U87 cells treated with AS-miR-221/222 and different dose of TMZ by MTT. All data are representative of $\geq 3$ independent experiments. Value is presented as means \pm SE. (C) Glioma cells were transfected pcDNA-PUMA (not including the 3'UTR), and PUMA expression was measured by Western blot assay. GAPDH protein was regarded as endogenous normalizer. (D) Relative proliferation of LN308, U251 and U87 cells treated with pcDNA-PUMA and different dose of TMZ by MTT.

than that needed to induce cytotoxicity with AS-miR-221/222 alone, and was more remarkable in the U251 cells than in the LN308 and U87 cells. The combination index values at $\mathrm{IC}_{50}$ for LN308, U251 and U87 treated with TMZ in combination with AS-miR-221/222 were 13.1, 7.5 and $13.6 \mu \mathrm{M}$, respectively.

Next, we evaluated whether expression of PUMA can increase the cytotoxicity of TMZ as well as downregulation of miR-221/222. After transfected by pcDNA-PUMA, the expression of PUMA was significantly increased in the glioma cells (Fig. 4C). Furthermore, we found that PUMA induced potentiation of the effects of TMZ (Fig. 4D). Accordingly, low expression of miR-221/222 increases TMZ-induced cytotoxicity in part by the direct post-transcriptional upregulation of PUMA.

To delineate the sensitization of AS-miR-221/222 to TMZ in glioma, we used Annexin V and caspase-3/7 activity. The results demostrated that combined treatment with AS-miR221/222 and TMZ remarkably increased the number of Annexin V-positive and PI-negative cells (Fig. 5A). Alternatively, the caspase-3/7 activity showed similar results (Fig. 5B). Moreover, 
A
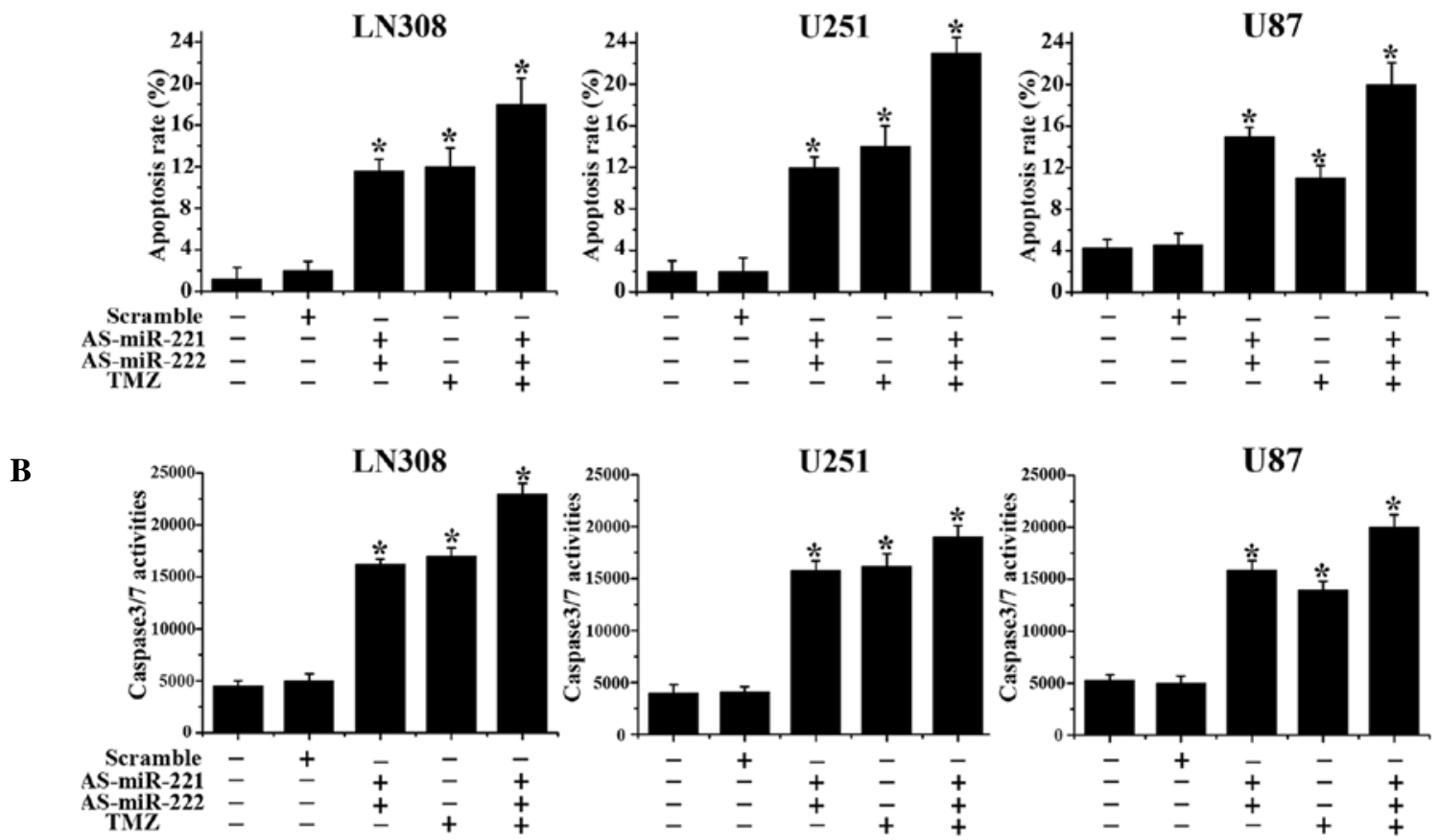

C
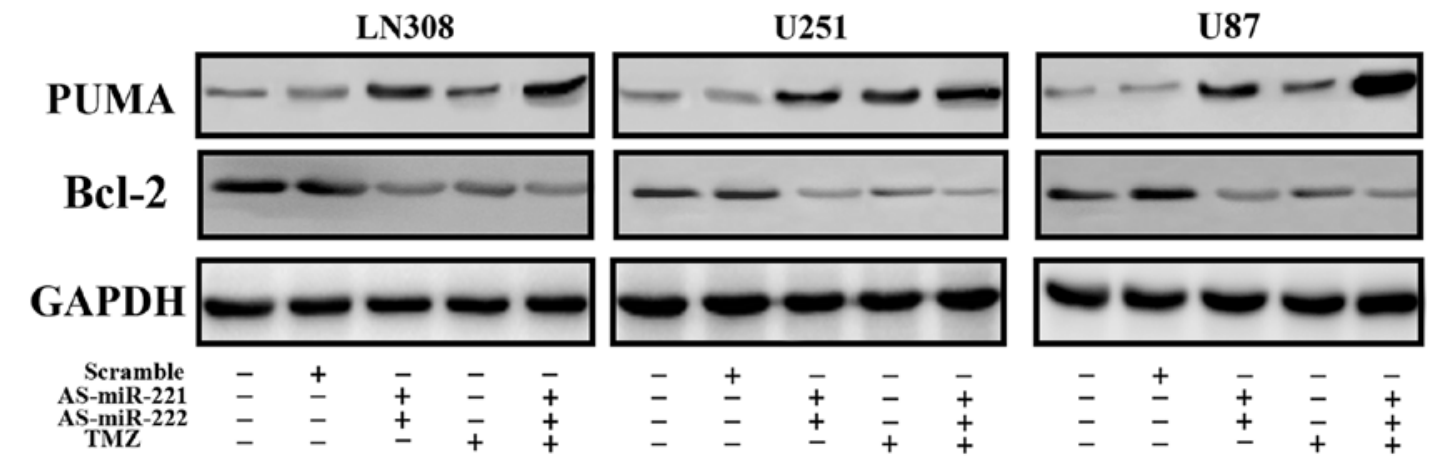

Figure 5. AS-miR-221/222 sensitize TMZ in glioma cells. (A) Annexin V analysis showed that LN308, U251 and U87 cells treated with As-miR-221/222 and TMZ displayed significantly more apoptosis than the other groups. (B) A significant increase in caspase-3/7 activity was detected in LN308, U251 and U87 cells treated with As-miR-221/222 and TMZ. (C) The effectiveness of AS-miR-221/222 combined with TMZ on PUMA and Bcl-2 were analyzed by Western blotting. Antibodies against GAPDH were used as a loading control.

Western blot assay displayed that pro-apoptotic protein PUMA expression was significantly upregulated while Bcl-2 was down-regulated in combination group compared with other groups (Fig. 5C).

\section{Discussion}

In the past few years, plenty of articles have been published describing a link between several forms of human cancer and the expression of microRNAs (13). Here, we focused on a pair of microRNAs, miR-221 and miR-222, both of which cluster on chromosome Xp11.3 and have the same targets (14). Functional studies indicated a connection of miR-221/222 with cell cycle progression (15) by targeting p27 and p57 (16). Recently, Zhang et al demonstrated that miR-221/222 have an important role in the regulation of cell apoptosis by direct targeting of the pro-apoptotic molecule PUMA (11). PUMA is located upstream of p53 that plays a role in several cellular processes, including the cell cycle, response of cells to DNA damage, cell death, and cell differentiation. The study of Zhang et al did not refer to whether miR-221/222 could induce apoptosis in different p53 status, because the glioma cell line U251 and LN229 they used were both p53 mutant. Since different p53 status can influence the chemosensitivity of astrocytic glioma cells, we chose three different p53 type glioma cell lines LN308 p53 null, U251 p53 mutant and U87 p53 wild-type. In each of them similar apoptosis could be induced with As-miR-221/222. Furthermore, with the employment of a specific siRNA construct against p53, no attenuation of apoptosis was found in p53 wild-type U87 cells after treatment by miR-221/222 inhibitor. In addition, Western blot assay showed As-miR-221/222 treated cells could increase the expression of Bax, cytochrome C, Apaf-1 and cleavedcaspase- 3 and decrease the expression of the Bcl-2, which are pivotal components of the intrinsic apoptosis pathway. Thus, we conclude that downregulation of miR-221/222 increases apoptosis independent of p53 status.

PUMA, a pro-apoptotic protein belongs to the BH3-only subgroup of Bcl-2 family, which induces apoptosis when transactivated by $\mathrm{p} 53$, in response to genotoxic stresses such as 
DNA damage (17). PUMA can also initiate p53-independent apoptotic responses, when activated by other transcription factors in response to non-genotoxic stimuli, including growth factor/cytokine deprivation, endoplasmic reticulum (ER) stress and ischemia/reperfusion (18). After exposure to genotoxic stress and non-genotoxic stimuli, the proapoptotic activity of PUMA requires its interactions with other $\mathrm{Bcl}-2$ family members and mitochondria localization. Biochemical studies indicated that PUMA induced apoptosis by activating the multidomain proapoptotic protein Bax and/or Bak through its interaction with anti-apoptotic Bcl-2 family members, thereby triggering mitochondrial dysfunction and caspase activation. Ito et al demostrated that overexpression of PUMA can lead to caspase- 3 activation regardless of p53 status in malignant glioma cells (19). In our study, luciferase assay verified that PUMA is the direct target of miR-221/222 in LN308, U251 and U87 cells which is consistent with previous studies. Ectopic expression of PUMA induced potentiation of the effects of TMZ in glioma cells and was more apparent in U251 than LN308 and U87 cells.

So far, the most effective chemotherapeutic for GBM is the DNA alkylating agent TMZ. Stupp et al (1) observed in newly diagnosed cases that the addition of TMZ chemotherapy to radiotherapy (RT) significantly prolonged tumor control and patient survival. However, not all patients with GBM respond to this DNA methylating agent (20). Blough et al delineated that traditional glioma cell lines that did not express a functional p53 were significantly more sensitive to TMZ than cell lines with functionally intact wild-type p53 expression. RNAi specific for p53 had little effect on sensitivity in p53 null glioma cells (21). Thus, we examined whether low expression of miR-221/222 can sensitize the effects of TMZ in different p53 status glioma cells. We found that the proliferation of glioma cells treated with TMZ was significantly suppressed with the miR-221/222 inhibitor. The AS-miR-221/222-induced potentiation of the effects of TMZ was recognized at a lower concentration than that needed to induce cytotoxicity with AS-miR-221/222 alone. In addition, combination of AS-miR221/222 with TMZ increased apoptosis in LN308, U251 and U87 cells as verified by Annexin assay and caspase-3/7 activity. Western blot analysis showed that the expression of PUMA was upregulated while $\mathrm{Bcl}-2$ was downregulated in combination group. Accordingly, our study demostrated that low expression miR-221/222 can sensitize TMZ in glioma independent of p53 staus. Taken together, downregulation of miR-221/222 is a potential treatment for combining with TMZ.

In summary, our data demonstrated that downregulation of miR-221/222 induced apoptosis in different p53 type cell lines. PUMA is the direct target of miR-221/222. In addition, low expression of miR-221/222 can sensitize TMZ in glioma of different p53 status.

\section{Acknowledgements}

This study was supported by the China National Natural Scientific Fund (30772231), and Tianjin City High School Science and Technology Fund (07ZCGHHZ01000).

\section{References}

1. Stupp R, Mason WP, van den Bent MJ, et al: Radiotherapy plus concomitant and adjuvant temozolomide for glioblastoma. $\mathrm{N}$ Engl J Med 352: 987-996, 2005.

2 Gabayan AJ, Green SB, Sanan A, et al: GliaSite brachytherapy for treatment of recurrent malignant gliomas: a retrospective multi-institutional analysis. Neurosurgery 58: 701-709, 2006.

3. Lasky JL III, Choe M and Nakano I: Cancer stem cells in pediatric brain tumors. Curr Stem Cell Res Ther 4: 298-305, 2009.

4. Besch R, Poeck H, Hohenauer T, et al: Proapoptotic signaling induced by RIG-I and MDA-5 results in type I interferonindependent apoptosis in human melanoma cells. J Clin Invest 119: 2399-2411, 2009.

5. Ciafre SA, Galardi S, Mangiola A, et al: Extensive modulation of a set of microRNAs in primary glioblastoma. Biochem Biophys Res Commun 334: 1351-1358, 2009.

6. He H, Jazdzewski K, Li W, et al: The role of microRNA genes in papillary thyroid carcinoma. Proc Natl Acad Sci USA 102: 19075-19080, 2005.

7. Ventura A and Jacks T: MicroRNAs and cancer: short RNAs go a long way. Cell 136: 586-591, 2009.

8. Zhou X, Ren Y, Moore L, et al: Downregulation of miR-21 inhibits EGFR pathway and suppresses the growth of human glioblastoma cells independent of PTEN status. Lab Invest 90: 144-155, 2010.

9. Zhang J, Han L, Ge Y, et al: miR-221/222 promote malignant progression of glioma through activation of the Akt pathway. Int J Oncol 36: 913-920, 2010.

10. Zhang C, Kang C, You Y, et al: Co-suppression of miR-221/222 cluster suppresses human glioma cell growth by targeting p27kip1 in vitro and in vivo. Int J Oncol 34: 1653-1660, 2009.

11. Zhang CZ, Zhang JX, Zhang AL, et al: MiR-221 and miR-222 target PUMA to induce cell survival in glioblastoma. Mol Cancer 9: 229, 2010

12. Yu J, Zhang L, Hwang PM, Kinzler KW and Vogelstein B: PUMA induces the rapid apoptosis of colorectal cancer cells. Mol Cell 7: 673-682, 2001.

13. Wu M, Jolicoeur N, Li Z, et al: Genetic variations of microRNAs in human cancer and their effects on the expression of miRNAs. Carcinogenesis 29: 1710-1716, 2008.

14. Galardi S, Mercatelli N, Giorda E, et al: miR-221 and miR-222 expression affects the proliferation potential of human prostate carcinoma cell lines by targeting p27Kip1. J Biol Chem 282: 23716-23724, 2007.

15. Visone R, Russo L, Pallante P, et al: MicroRNAs (miR)-221 and miR-222, both overexpressed in human thyroid papillary carcinomas, regulate p27Kip1 protein levels and cell cycle. Endocr Relat Cancer 14: 791-798, 2007.

16. Medina R, Zaidi SK, Liu CG, et al: MicroRNAs 221 and 222 bypass quiescence and compromise cell survival. Cancer Res 68: 2773-2780, 2008.

17. Yu J and Zhang L: No PUMA, no death: implications for p53-dependent apoptosis. Cancer Cell 4: 248-249, 2003.

18. Yu J and Zhang L: PUMA, a potent killer with or without $\mathrm{p} 53$. Oncogene 27 (Suppl 1): S71-S83, 2008.

19. Ito H, Kanzawa T, Miyoshi T, et al: Therapeutic efficacy of PUMA for malignant glioma cells regardless of p53 status. Hum Gene Ther 16: 685-698, 2005.

20. Hegi ME, Diserens AC, Gorlia T, et al: MGMT gene silencing and benefit from temozolomide in glioblastoma. N Engl J Med 352: 997-1003, 2005.

21. Blough MD, Beauchamp DC, Westgate MR, Kelly JJ and Cairncross JG: Effect of aberrant p53 function on temozolomide sensitivity of glioma cell lines and brain tumor initiating cells from glioblastoma. J Neurooncol 102: 1-7, 2011. 